\title{
Article
}

\section{D printed oral theophylline doses with innovative 'radiator-like' design: Impact of polyethylene oxide (PEO) molecular weight}

Isreb, Abdullah, Baj, Krzysztof, Wojsz, Magdalena, Isreb, Mohammad, Peak, Matthew and Alhnan, Mohamed A

Available at http://clok.uclan.ac.uk/28342/

Isreb, Abdullah ORCID: 0000-0001-9939-6161, Baj, Krzysztof, Wojsz, Magdalena, Isreb, Mohammad, Peak, Matthew and Alhnan, Mohamed A (2019) 3D printed oral theophylline doses with innovative 'radiator-like' design: Impact of polyethylene oxide (PEO) molecular weight. International Journal of Pharmaceutics, 564 . pp. 98-105. ISSN 0378-5173

It is advisable to refer to the publisher's version if you intend to cite from the work. http://dx.doi.org/10.1016/j.jpharm.2019.04.017

For more information about UCLan's research in this area go to http://www.uclan.ac.uk/researchgroups/ and search for <name of research Group>.

For information about Research generally at UCLan please go to http://www.uclan.ac.uk/research/

All outputs in CLoK are protected by Intellectual Property Rights law, including Copyright law. Copyright, IPR and Moral Rights for the works on this site are retained by the individual authors and/or other copyright owners. Terms and conditions for use of this material are defined in the policies page. 


\section{3D Printed Oral Theophylline Doses with Innovative}

2 'Radiator-Like' Design: Impact of Polyethylene Oxide (PEO)

3 Molecular Weight

4

5

6 Abdullah Isreb ${ }^{\mathrm{a}}$, Krzysztof Baj ${ }^{\mathrm{b}}$, Magdalena Wojsz $^{\mathrm{c}}$, Mohammad Isreb ${ }^{\mathrm{d}}$, Matthew Peak $^{\mathrm{e}}$, 7 Mohamed A Alhnan ${ }^{\mathrm{f}} *$

8

$9{ }^{a}$ School of Pharmacy and Biomedical Sciences, University of Central Lancashire, Preston, 10 Lancashire, $U K$

$11{ }^{b}$ Faculty of Pharmacy, Medical University of Lodz, Lodz, Poland

$12{ }^{\mathrm{c}}$ Faculty of Pharmacy with the Laboratory Medicine Division, Medical University of Warsaw,

13 Warsaw, Poland

$14{ }^{\mathrm{d}}$ School of Pharmacy, University of Bradford, Richmond Road, Bradford, UK

$15{ }^{e}$ Paediatric Medicines Research Unit, Alder Hey Children's NHS Foundation Trust, Liverpool, UK

$16{ }^{f}$ Institute of Pharmaceutical Science, King's College London, London, UK 
Despite the abundant use of polyethylene oxides (PEOs) and their integration as an excipient in numerous pharmaceutical products, there have been no previous reports of applying this important thermoplastic polymer species alone to fused deposition modelling (FDM) 3D printing. In this work, we have investigated the manufacture of oral doses via FDM 3D printing by employing PEOs as a backbone polymer in combination with polyethylene glycol (PEG). Blends of PEO (molecular weight $100 \mathrm{~K}, 200 \mathrm{~K}, 300 \mathrm{~K}, 600 \mathrm{~K}$ or $900 \mathrm{~K}$ ) with PEG $6 \mathrm{~K}$ (plasticiser) and a model drug (theophylline) were hot-melt extruded. The resultant filaments were used as a feed for FDM 3D printer to fabricate oral dosage forms (ODFs) with innovative designs. ODFs were designed in a radiator-like geometry with connected paralleled plates and inter-plate spacing of either $0.5,1,1.5$ or $2 \mathrm{~mm}$. X-ray diffraction patterns of the filaments revealed the presence of two distinctive peaks at $2 \theta=7^{\circ}$ and $12^{\circ}$, which can be correlated to the diffraction pattern of theophylline crystals. Varying blends of PEO and PEG allowed the formation of mechanically resistant filaments (maximum load at break of 357, 608, 649, 882, $781 \mathrm{~N}$ for filament produced with PEO 100K, 200K, 300K, $600 \mathrm{~K}$ or $900 \mathrm{~K}$, respectively). Filaments of PEO at a molecular weight of $200-600 \mathrm{~K}$ were compatible with FDM 3D printing process. Further increase in PEO molecular weight resulted in elevated shear viscosity $\left(>10^{4}\right.$ $\mathrm{Pa} . \mathrm{S})$ at the printing temperature and hindered material flow during FDM 3D printing process. A minimal spacing $(1 \mathrm{~mm})$ between parallel plates of the radiator-like design deemed essential to boost drug release from the structure. This is the first report of utilising this widely used biodegradable polymer species (PEOs and PEG) in FDM 3D printing.

A R T I C L E I N F O

Keywords:

Personalised medicine, additive manufacturing, complex structures, tablets, patient-specific, structural 


\section{Introduction}

Through recent advances in pharmacogenetics the relationship between an individual's genome, their genetic predisposition to disease and their response to specific medications is increasingly understood [1]. With an increased focus on patient-centred and stratified treatment, there is a growing need for a technological solution to provide individual patients with reliable and safe personalised dosage forms. In the last few years, additive manufacturing has been proposed as alternative platform for on-demand production of personalised dosage forms with significant ability to tailor the size, shape, dose as well as drug release pattern [24].

Among other commercially available technologies, fused deposition modelling (FDM) 3D printing offers major advantages, including the low cost of the printer, the absence of finishing steps and the lack need for powder facilities. These properties position FDM 3D printing as a very attractive platform for small-scale individualising for solid dosage forms. Recently, several examples of the use of FDM 3D printing for production of immediate, delayed and extended drug release have been reported [3, 5-9]. The technology proved efficacy at accurately titrating coumarin doses in animals [10] and extended drug release in gastro-retentive systems [11].

For the pharmaceutical industry to make a full use of 3D printing, it is essential to adapt pharmaceutical grade polymers for FDM 3D printing. Previous studies have used cellulose, methacrylate, acrylic acid or PVP derivatives to produce solid dosage forms [12]. PEO is one of the most commonly used polymers in pharmaceutical industry. PEO is commercially available between $100 \mathrm{~K}$ to $10,000 \mathrm{~K} \mathrm{~g}$ /mole and has been extensively used for oral and parental formulations (Gullapalli and Mazzitelli, 2015). PEOs have been commonly used to produce extended release tablets in powder compression Moroni and Ghebresellassie, 1995), hot melt extrusion (Zhang and McGinity, 1999) and in buccal tablets (Apicella et al., 1993). However, limited reports are available applying this extensively used polymer species to FDM 3D printing. In rare examples, PEO was used for formation of thin oral film in combination with other additives [13], or as an additive to methacrylate polymer for 3D printing of tablets [14].

In order for a filament to be compatible with the FDM 3D printing process, it requires critical mechanical and rheological criteria [15]. Previous studies have linked a filament's 3D printing compatibility with the rheological properties of the backbone polymers: poly methacrylate [8], PVA [16] and PVP-VA [16, 17]. The availability of PEOs at different molecular weight grades 
provides the opportunity to test the impact of polymeric molecular weight and rheological flow properties of a single polymer.

In this work, we have investigated the fabrication of oral doses via FDM 3D printing by employing PEOs as a backbone polymer in combination with PEG as a plasticiser. We assessed the impact of polymer molecular weight on the mechanical properties of the resultant filaments and their rheological properties. We have also tested the effect of an innovative radiator-like design of the solid dosage form on the acceleration of drug release patterns.

\section{Materials and Methods}

\subsection{Materials}

Theophylline was supplied by Acros Organics (UK). Polyethylene glycol (PEG 6000) and all grades of polyethylene oxide (PEO) were purchased from Sigma-Aldrich (Dorset, UK).

\subsection{Preparation of filaments using hot melt extrusion (HME)}

Filaments were prepared by mixing polyethylene oxide (PEO molecular weight of 100K, 200K, 300K, 600K, or 900K), Polyethylene Glycol (PEG 6K) and theophylline (Table 1). The mixtures were extruded using a Thermo Scientific HAAKE MiniCTW hot melt extruder (Karlsruhe, Germany) after mixing inside the extruder for $5 \mathrm{~min}$ at a temperature range of 60$80^{\circ} \mathrm{C}$ (Table1) at $35 \mathrm{rpm}$ using $1.5 \mathrm{~mm}$ nozzle.

\subsection{Tablet design and printing}

Tablets were designed using Autodesk ${ }^{\circledR}$ 3ds Max Design 2016 software version 18.0 (Autodesk, Inc., USA). In the CAD design, the radiator-like tablets were structured with increasing inter-plate spacing of 5, 10, 15 or $20 \mathrm{~mm}$ whilst the overall dimensions of the design were maintained within the volume of $20 \times 10 \times 6 \mathrm{~mm}$. The templates were then imported into the $3 \mathrm{D}$ printer software in a stereolithography (.stl) file format. The previously extruded filaments were fed into the FDM 3D printer equipped with $0.4 \mathrm{~mm}$ nozzle size and MakerWare Version 2.4.0.17 (Makerbot Industries, LLC, USA). Tablets were printed using modified settings of the software as described earlier in our previous work [18]:Replicator 2X; type of filament: PLA; resolution: standard; temperature of building plate: $40{ }^{\circ} \mathrm{C}$; speed of extruder $50 \mathrm{~mm} / \mathrm{sec}$ while extruding and $150 \mathrm{~mm} / \mathrm{sec}$ while traveling; infill: 100\%; height of the layer: $200 \mu \mathrm{m}$. The temperature of the nozzle for each filament is specified in Table 1.

\subsection{Thermal analysis}


Thermal decomposition profiles for PEOs as both received and extruded filaments were measured using a TA Q500 Thermogravimetric Analyzer TGA (TA Instruments, Elstree, Hertfordshire, UK). Samples with an average weight of $10 \mathrm{mg}$ were measured from $25^{\circ} \mathrm{C}$ to $500^{\circ} \mathrm{C}$ with a heating rate of $10^{\circ} \mathrm{C} / \mathrm{min}$ and a nitrogen gas purge of $40 / 60 \mathrm{~mL} / \mathrm{min}$ for sample/furnace respectively. The thermal behaviour of these samples was measured using a TA Q2000 Differential Scanning Calorimeter (DSC) (TA Instruments, Elstree, Hertfordshire, UK). Samples ( $5 \mathrm{mg}$ ) were prepared in aluminium standard pans $(40 \mu \mathrm{L})$ and sealed with pinholed lid. Each sample was heated from -10 to $255^{\circ} \mathrm{C}$ at $10^{\circ} \mathrm{C} / \mathrm{min}$ under a nitrogen purge of $50 \mathrm{~mL} / \mathrm{min}$. Data from TGA and DSC were analysed using a TA 2000 analysis software (TA Instruments, Elstree, Hertfordshire, UK). All measurements were carried out in triplicate.

\subsection{X-ray Powder diffractometry (XRPD)}

An X-ray powder diffractometer, D2 Phaser with Lynxeye (Bruker, Germany) was used to assess the physical form of theophylline, PEO, PEG and drug loaded filaments. Samples were scanned from $(2 \theta)=5^{\circ}$ to $50^{\circ}$ using $0.01^{\circ}$ step width and a 1 second time count. The X-ray wavelength of $0.154 \mathrm{~nm}$ was used using a $\mathrm{Cu}$ source and a voltage of $30 \mathrm{Kv}$. The divergence slit was $1 \mathrm{~mm}$ and the scatter slit $0.6 \mathrm{~mm}$. Filament emission was $10 \mathrm{~mA}$ using a scan type coupled with a two theta/theta scintillation counter over $60 \mathrm{~min}$.

\subsection{Hansen solubility parameter}

Hansen solubility parameters for the polymer and the drugs were calculated using HSPiP software (version 5.0.08).

\subsection{Scanning electron microscopy (SEM)}

The topography of the drug-loaded filaments and the 3D printed tablets were examined using Quanta-200 SEM microscope at $20 \mathrm{kV}$. Samples were coated under vacuum with a gold coater JFC-1200 Fine Coater (Jeol, Tokyo, Japan). In addition, photographs of tablets were collected a Canon EOS-1D Mark IV (Canon Ltd, Japan).

\subsection{Rheology studies}

A shear Physica MCR 501 rheometer (Anton Paar, Germany) was used in oscillation mode with a parallel plate configuration (plate diameter $=25 \mathrm{~mm}$ ). The gap between the plate and the base was set at $0.5 \mathrm{~mm}$. Amplitude sweep test was performed to determine the linear 
viscoelastic region (LVR). Afterwards, frequency sweep tests were performed at a strain amplitude of $1 \%$ (Well within the LVR region) and an angular frequency range from 100 to $0.1 \mathrm{rad} / \mathrm{sec}$. Each sample was tested at three temperatures; 100,110 and $140^{\circ} \mathrm{C}$. The readings $(n=6)$ were recorded for each frequency decade (18 points in total). The test was only carried out after the normal force recorded by the device dropped below $1 \mathrm{~N}$, which indicates that the polymer is in a relaxed state. Power law fit was used in the linear shear thinning area of the obtained rheological data to measure the shear-thinning index $(n)$. Elastic $\left(\mathrm{G}^{\prime}\right)$ and viscous (G”) moduli as well as complex viscosity data were recorded and plotted against the angular frequency at each temperature.

\subsection{Tensile strength studies}

A tensile strength testing system 5568 (Instron, Buckinghamshire, UK) was used to measure the breaking stress for filaments with irregular geometry with an average diameter of approximately 1.8 and $10 \mathrm{~mm}$ gauge length. The diameter of the samples was measured using a Vernier micro-caliper for various sections and the average (c.a. $1.8 \mathrm{~mm}$ ) was programmed into the software. The deformation rate (extension) was set to $20 \mathrm{~mm} / \mathrm{min}$ and the data were collected every $50 \mathrm{msec}$. A sand paper was used to prevent the slipping of the sample from the clamp. Samples that showed signs of slipping from the clamp were rejected and all samples were measured in triplicate. A stress strain graph was plotted for each sample and the breaking stress was measured.

\subsection{Drug Contents and in vitro drug release studies}

For assessment of theophylline contents, oral doses were dissolved in $500 \mathrm{~mL}$ of deionised water and were stirred consciously for one hour at $40{ }^{\circ} \mathrm{C}$ until complete dissolution. Samples were filtered through a $0.22 \mu \mathrm{m}$ Millex-GP syringe filter (Merck Millipore, USA) and the concentration of the drug was determined using a Jenway Spectrophotometer (Bibby Scientific Ltd, UK) at $\lambda$ max of $272 \mathrm{~nm}(\mathrm{n}=3)$.

To study in vitro theophylline release for 3D printed tablets, An AT 7 Smart USP II dissolution test apparatus (Sotax, Switzerland) was used. A dissolution medium of $900 \mathrm{~mL} \mathrm{0.1M} \mathrm{HCl} \mathrm{(pH} \mathrm{1.2)} \mathrm{at}$

$16237 \pm 0.5^{\circ} \mathrm{C}$ with a paddle speed of $50 \mathrm{rpm}$ was used for 2 hours. Each experiment was carried out in

163 triplicate. Samples were collected at $5 \mathrm{~min}$ intervals and drug concentration was determined using 164 UV/VIS spectrophotometer (PG Instruments Limited, UK) at the wavelength of $272 \mathrm{~nm}$ and path length of $10 \mathrm{~mm}$ and outcome data were analysed using IDISis software 2012 (Automated Lab, UK).

\subsection{Statistical analysis}


167 The data were analysed by one-way ANOVA using SPSS Software (22.0.0.2). The level of 168 attributed significance for comparisons were as follows: $p>0.05$ not significant; $p \leq 0.05$ 169 significant.

170 


\section{Results and discussion}

172 Polyethylene glycol (PEG) and polyethylene oxide (PEO) are two of the most widely used excipients in pharmaceutical products. Both products are also used in other healthcare applications. Both polymers are also biodegradable and suitable to be used as a polymeric biomaterial in tissue scaffolding [19]. PEGs are considered a safe choice to prepare hydrogel sealant for patients undergoing surgery [20] and are also used in the manufacturing of 3D porous scaffolds [21]. Optimisation of pharmaceutical solid dosage forms produced by FDM 3D printing requires a suitable and compatible polymer backbone for the feed filament. Initially, PEGs were first assessed producing feed filaments for FDM 3D printing (as a backbone polymer). However, the hot melt extrusion process only yielded easily breakable PEGbased filaments which lacked the required rheological and mechanical properties to enable for the use of PEGs in FDM 3D printing of solid dosage forms (data not shown). Therefore, a higher molecular weight thermoplastic polymer (PEO), was used for its mechanical and rheological properties while PEG was added as a plasticiser to facilitate the material flow and pore former to accelerate drug release from the dosage form produced by FDM 3D printing.

The thermal properties of PEOs of different molecular weights $(100 \mathrm{~K}-900 \mathrm{~K})$ were shown to be stable at $<150^{\circ} \mathrm{C}$ (Fig. 1A). In addition, PEO revealed a minimum moisture content with a weight loss of $<2 \%$ at $120^{\circ} \mathrm{C}$. The polymer showed no significant change in thermal degradation following the compounding into a filament with the addition of PEG and theophylline via HME extrusion (Fig. 1B). Pourepolymer melting was observed above $66-69^{\circ} \mathrm{C}$ (data not shown) [22]. However, the compounded filament produced in this study showed slightly lower melting points (in the range of $62-65.9^{\circ} \mathrm{C}$ ), which could be attributed to the addition of a lower melting point additive (PEG) (Fig. 1C). Thermal profiles also illustrated that theophylline was crystalline within the polymer matrix with the appearance of theophylline melting endotherm known to be at $\sim 240^{\circ} \mathrm{C}[23]$.

XRD patterns confirmed the crystallinity status of PEO 200K and PEG 6K with the presence of intensity peaks at $2 \theta=19.1^{\circ}$ and $23.2^{\circ}$ the appearance of these peaks in the pattern of HME compounded filament suggests that polymers remained crystalline. The diffraction patterns of extruded filaments also revealed diffraction peaks at $2 \theta=7^{\circ}$ and $12.9^{\circ}$ (Fig. 3). The later peaks are characteristic peaks in the diffraction pattern of theophylline [7]. This confirms the crystalline structure of theophylline within the polymeric matrix. The diffraction patterns of filaments produced with other molecular weight PEOs $(100 \mathrm{~K}, 300 \mathrm{~K}$, $600 \mathrm{~K}$ and $900 \mathrm{~K}$ ), also revealed the presence of crystalline theophylline (Supplementary data, Figs. S14).

The Hansen solubility parameter data of the PEO and PEG blend and the drug are shown in Table 2. The difference in solubility parameter between the PEO and PEG blend and the drug $\left(\Delta \delta=7 \mathrm{MPa}^{1 / 2}\right)$, indicated a minimal miscibility between these molecules and predicted the presence of theophylline as a solid suspension within PEG/PEO polymeric matrix. 
The impact of molecular weight on mechanical properties of HME compounded filaments was assessed using the tensile strength test (Fig. 3A). HME compounded filaments including PEO of 100K molecular weight showed the least maximum load before break $(357 \mathrm{~N})(p<0.05)$ and were deemed too fragile. As the filament breaks instantly upon the application of gear pressure in the FDM 3D printer's head. HME compounded filaments including PEO of $200 \mathrm{~K}$ molecular weight were able to be loaded through the gears of the FDM 3D printer head. However, frequent breakage of the filament due to the pressure of the gears interrupted the printing process and resulted in printing failure. When HME compounded filaments containing PEO of higher molecular weight (300K, 600K and 900K), the filaments were able to withstand higher tension (Fig. 3A). The maximum load at break steadily increased with longer polymer chains [24]. On the other hand, Young modulus of PEO 100K based filament reveal more brittle behaviour in comparison to filaments produced with higher molecular weight PEO (Fig. 3B). The increased plasticity of HME compounded filaments containing higher molecular weight PEO also allows the filament to withstand more pressure from the gears of the head of the FDM 3D printer and mitigates the risk of filament breakage. This increase in the strength of the filament can be related to previous observations of the reduced mobility due to the entanglement of the amorphous parts of the polymeric chains associated with an increase in the chain length [25]

During the FDM 3D printing process, the filament passed through lead to a hot channel that terminates in a nozzle and while the path is narrowed from 1.75 to $0.4 \mathrm{~mm}$ (nozzle diameter), the filament experiences an increase from room temperature to the printing temperature $\left(110-145^{\circ} \mathrm{C}\right)$. Therefore, it is essential to study the rheological behaviour of the filament compositions at the temperature of the printing nozzle. Hence, complex viscosity under various angular frequency at two representative printing temperatures $\left(110\right.$ and $145^{\circ} \mathrm{C}$ ) were performed (Fig. 4). Complex viscosity of a polymer is a temperature-dependent material property [26]. Despite the similarity of the melting points across all PEO grades, the printability of each filament using FDM 3D printing was dependent on the temperature of the 3D printer temperature (Table 1). The lower complex viscosities of PEO 100K (539.8 Pa.S) and 200K (1385.31 Pa.S) based filaments suggest possible flow from the hot nozzle of the 3D printer (Fig. 5). However, it was not possible to physically test 3D printing using these filaments due to their incompatibility with the gears of the 3D printer's head (see above). However, HME compounded filaments including PEO of higher molecular weights allowed consistent flow from the hot nozzle at a printing temperature of 110 and $145^{\circ} \mathrm{C}$ for PEO 300K and 600K respectively (Fig. 5). The complex viscosity of these filaments was in the range of 9000 and 10000 at the corresponding temperature at $1 \%$ angular viscosity. Filament containing higher molecular weight PEO (900K) was observed to have a high complex viscosity (>22610 Pa.S) and was associated with restricted materials flow in the nozzle of the 3D printer and obstructed the printing of this particular filament. Further increase in nozzle temperatures (up to $220^{\circ} \mathrm{C}$ ), did not improve material flow of this specific HME compounded filament. This may be because increasing temperature above $150{ }^{\circ} \mathrm{C}$ is likely to accelerate PEO degradation [27]. 
242 It can be deduced that a complex viscosity of approximately $<8000 \mathrm{~Pa} . \mathrm{S}$ is necessary to achieve 243 sufficient material flow from the FDM 3D printer hot nozzle and successful completion of FDM 3D 244 printing.

245 The viscoelastic properties of the filaments produced with PEOs of different MW were characterised 246 through the measurement of the storage $G^{\prime}$ and loss modulus $G^{\prime \prime}$ (Fig. 6). In general, increasing the 247 temperature led to a decrease in both storage modulus $G^{`}$ and loss modulus $G^{\prime \prime}$ across different 248 molecular weights. Filaments containing PEO 100K were noticed to be in a terminal flow zone as $249 \mathrm{G}^{\prime}>\mathrm{G}^{\prime}$. A higher PEO molecular weight in the filament resulted in less liquid-like flow and a more elastic behaviour as the polymer was approaching crossover point. Following extrusion from the nozzle of the 3D printer, the filament loses its microstructure and conforms to the architecture dictated by the CAD design and slicing engine. This behaviour can be advantageous in FDM 3D printing as it provides a wide variety of molecular weights to select from while maintaining the same release profile. This observation needs to be repeated and validated with other drugs that may interact with PEO.

Unlike regular caplet design, where filaments are not only fused with lower and upper layers, but also with side printed layers, the radiator-like design only allows fusion with upper and lower layers, leading to potentially different mechanical behaviour to solid caplet design. However, it was not possible to measure the tensile strength of oral doses due to their thin structure, where weak clamping point of the structures deemed it unsuitable for the test.

When theophylline release from capsule-shaped tablets with PEO 600K produced by FDM 3D printing was assessed (Supplementary data, Fig. S2), a slow release profile was observed. It is likely that the drug is released though erosion of the polymeric matrix and diffusion mechanisms [28]. The polymerrich structure of the caplet hindered drug release. The fast hydration of PEO/PEG based tablets produced by powder compression was reported to yield a gel-layer upon introduction to dissolution medium that significantly prolongs drug release [29]. In fact, PEO/PEG blends have been devised to produce tablet with extended release over 12-24 hours [30]. In such matrix systems, drug release is dependent on the rate of polymer dissolution [31], which regulates the pattern of drug release and often yields a zero order pattern [32].

In order to accelerate drug release from PEO matrix, an alternative novel design approach of a radiatorlike architecture was evaluated (Fig. 7). The proposed geometry allows 7-8-fold increase in surface-tomass ratio of the structure (Table 3). Moreover, the design facilitates water penetration and drug permeation from the PEO matrix by minimizing the thickness of gel-layer with the use of low-thickness plates. Four designs with identical overall dimensions but with increasing spaces $(0.5,1.0,1.5$ and 2.0 $\mathrm{mm}$ ) between the design plates were tested. With increasing inter-plate spacing within the dimensions $20 \times 10 \times 6 \mathrm{~mm}$, the number of plates has decreased and resulted in lower printed mass and dose (Fig. 7, Table 3). A minimum spacing of $1 \mathrm{~mm}$ was deemed essential to accelerate drug release from the 
structure and meet USP criteria for immediate release products (Fig. 8A). Similar drug release was obtained within the FDM 3D-printable range of PEOs (200K-600K) (Fig. 8B). Following introduction to the dissolution apparatus, the PEO matrix hydrates and swells leading to significant growth in the thickness of the radiator plate. Despite similar surface-to-mass ratio of these oral dose designs (Table 3 ), the $0.5 \mathrm{~mm}$ spaced design appeared to be slower in comparison with the rest of designs. It is possible that such swelling in the $0.5 \mathrm{~mm}$-spaced design resulted in plate adhesion, leading to reduction of contact surface area with the dissolution medium and hence slowing drug release. The paper provides a unique example of how 3D printing and novel design approach can significantly alter the release profile of the same formulation. The use of radiator-like design maximised interaction with dissolution medium and prevented the formation of thick permission gel layer, which will slow down drug release. In the future, such design approach will help to personalise the release profile without the need to modify the formulation.

\section{Conclusion}

This work demonstrates the effect of PEO molecular weight on the compatibility of HME compounded filaments for FDM 3D printing. A molecular weight of PEO between 300K-600K was shown to have optimal mechanical and rheological properties for the FDM 3D printing process. A lower molecular weight of PEO (100K-200K) yielded mechanically incompatible HME compounded filaments and a larger molecular weight of PEO (900K) contributed to significantly high complex viscosity and inhibited material flow. The use of a relatively low printing temperature $105-145^{\circ} \mathrm{C}$ potentially extends the applicability of this technology to a wider range of active pharmaceutical ingredients. A novel radiator-like paralleled plate geometry oral doses containing widely used biodegradable polymer species (PEOs and PEG) was reported. By using this architecture, it was possible to accelerate drug release and overcome polymer hindrance of theophylline release through PEO swelling and erosion. These findings are essential in the development of next-generation personalised drug delivery doses using specialised polymer/polymer blends purposely optimised for FDM 3D printing. 


\section{References}

305 1. Hamburg, M.A. and F.S. Collins, The path to personalized medicine. New England Journal of

2. Alhnan, M.A., et al., Emergence of 3D Printed Dosage Forms: Opportunities and Challenges. Pharm Res, 2016. 33(8): p. 1817-32.

3. Palo, M., et al., 3D printed drug delivery devices: perspectives and technical challenges. Expert Rev Med Devices, 2017. 14(9): p. 685-696.

4. Prasad, L.K. and H. Smyth, 3D Printing technologies for drug delivery: a review. Drug Dev Ind Pharm, 2016. 42(7): p. 1019-31.

5. Goyanes, A., et al., 3D printing of modified-release aminosalicylate (4-ASA and 5-ASA) tablets. Eur J Pharm Biopharm, 2015. 89: p. 157-62.

6. Tagami, T., et al., 3D Printing Factors Important for the Fabrication of Polyvinylalcohol Filament-Based Tablets. Biol Pharm Bull, 2017. 40(3): p. 357-364.

7. Pietrzak, K., A. Isreb, and M.A. Alhnan, A flexible-dose dispenser for immediate and extended release 3D printed tablets. Eur J Pharm Biopharm, 2015. 96: p. 380-7.

8. Sadia, M., et al., Adaptation of pharmaceutical excipients to FDM 3D printing for the fabrication of patient-tailored immediate release tablets. Int J Pharm, 2016. 513(1-2): p. 659668.

9. Skowyra, J., K. Pietrzak, and M.A. Alhnan, Fabrication of extended-release patient-tailored prednisolone tablets via fused deposition modelling (FDM) 3D printing. European Journal of Pharmaceutical Sciences, 2015. 68: p. 11-17.

10. Arafat, B., et al., Tailored on demand anti-coagulant dosing: an in vitro and in vivo evaluation of 3D printed purpose-designed oral dosage forms. Eur J Pharm Biopharm, 2018.

11. Li, Q., et al., Preparation and investigation of novel gastro-floating tablets with 3D extrusionbased printing. Int J Pharm, 2018. 535(1-2): p. 325-332.

12. Awad, A., et al., 3D printed medicines: A new branch of digital healthcare. Int J Pharm, 2018. 548(1): p. 586-596.

13. Ehtezazi, T., et al., The Application of 3D Printing in the Formulation of Multilayered Fast Dissolving Oral Films. J Pharm Sci, 2018. 107(4): p. 1076-1085.

14. Alhijjaj, M., P. Belton, and S. Qi, An investigation into the use of polymer blends to improve the printability of and regulate drug release from pharmaceutical solid dispersions prepared via fused deposition modeling (FDM) 3D printing. Eur J Pharm Biopharm, 2016. 108: p. 111125.

15. Nasereddin, J.M., et al., Development of a Simple Mechanical Screening Method for Predicting the Feedability of a Pharmaceutical FDM 3D Printing Filament. Pharm Res, 2018. 35(8): p. 151.

16. Boetker, J., et al., Modifying release characteristics from 3D printed drug-eluting products. Eur J Pharm Sci, 2016. 90: p. 47-52.

17. Fuenmayor, E., et al., Material Considerations for Fused-Filament Fabrication of Solid Dosage Forms. Pharmaceutics, 2018. 10(2).

18. Skowyra, J., K. Pietrzak, and M.A. Alhnan, Fabrication of extended-release patient-tailored prednisolone tablets via fused deposition modelling (FDM) 3D printing. Eur J Pharm Sci, 2015. 68: p. 11-17.

19. Bliley, J.M. and K.G. Marra, Polymeric biomaterials as tissue scaffolds, in Stem Cell Biology and Tissue Engineering in Dental Sciences. 2015, Elsevier. p. 149-161.

20. Cosgrove, G.R., et al., Safety and efficacy of a novel polyethylene glycol hydrogel sealant for watertight dural repair. Journal of neurosurgery, 2007. 106(1): p. 52-58.

21. Husár, B., et al., Photopolymerization-based additive manufacturing for the development of 3D porous scaffolds, in Biomaterials for Bone Regeneration. 2014, Elsevier. p. 149-201.

22. Beech, D.R. and C. Booth, Thermodynamic melting point of poly(ethylene oxide). Journal of Polymer Science Part B: Polymer Letters, 1970. 8(10): p. 731-734. 
23. Hock, C., et al., Melting-point depression by insoluble impurities: a finite size effect. Physical review letters, 2008. 101(2): p. 023401.

24. Husken, D. and R. Gaymans, The tensile properties of poly (ethylene oxide)-based segmented block copolymers in the dry and wet state. Journal of materials science, 2009. 44(10): p. 2656-2664.

25. Kennedy, M., A. Peacock, and L. Mandelkern, Tensile properties of crystalline polymers: linear polyethylene. Macromolecules, 1994. 27(19): p. 5297-5310.

26. Wissbrun, K.F., Observations on the melt rheology of thermotropic aromatic polyesters. British Polymer Journal, 1980. 12(4): p. 163-169.

27. Crowley, M.M., et al., Stability of polyethylene oxide in matrix tablets prepared by hot-melt extrusion. Biomaterials, 2002. 23(21): p. 4241-8.

28. Shojaee, S., et al., An Investigation on the Effect of Polyethylene Oxide Concentration and Particle Size in Modulating Theophylline Release from Tablet Matrices. AAPS PharmSciTech, 2015. 16(6): p. 1281-1289.

29. Maggi, L., R. Bruni, and U. Conte, High molecular weight polyethylene oxides (PEOS) as an alternative to HPMC in controlled release dosage forms. Int J Pharm, 2000. 195(1-2): p. 22938.

30. Kojima, H., et al., Extended release of a large amount of highly water-soluble diltiazem hydrochloride by utilizing counter polymer in polyethylene oxides (PEO)/polyethylene glycol (PEG) matrix tablets. Eur J Pharm Biopharm, 2008. 70(2): p. 556-62.

31. Apicella, A., et al., Poly(ethylene oxide) (PEO) and different molecular weight PEO blends monolithic devices for drug release. Biomaterials, 1993. 14(2): p. 83-90.

32. Kim, C.J., Effects of drug solubility, drug loading, and polymer molecular weight on drug release from Polyox tablets. Drug Dev Ind Pharm, 1998. 24(7): p. 645-51. 
Figure 1. TGA thermal decomposition profile of: A) raw PEO powder with molecular weights $(100 \mathrm{~K}, 200 \mathrm{~K}, 300 \mathrm{~K}, 600 \mathrm{~K}$, and $900 \mathrm{~K}), \mathrm{B})$ hot melt extruded filaments containing $30 \%$ theophylline, 35\% PEG 6K, and 35\% PEO (100K, 200K, 300K, 600K, and 900K), and C) DSC thermographs of corresponding filaments.

Figure 2. Representative XRD diffraction patterns of raw theophylline, PEG 6K, raw PEO $200 \mathrm{~K}$, and hot melt extruded filament containing 35\% PEO200K, 35\% PEG 6K, and 30\% theophylline (for other grades, see Figure S1 in Supplementary Data)

Figure 3. Tensile strength data of A) maximum load at break and B) Young Modulus for hot melt extruded filaments containing $30 \%$ theophylline, 35\% PEG 6K, and 35\% PEO (100K, $200 \mathrm{~K}, 300 \mathrm{~K}, 600 \mathrm{~K}$, and 900K).

Figure 4 Shear index for filaments containing $30 \%$ theophylline, 35\% PEG 6K, and 35\% PEO $(100 \mathrm{~K}, 200 \mathrm{~K}, 300 \mathrm{~K}, 600 \mathrm{~K}$, and $900 \mathrm{~K})$ at 110 and $145^{\circ} \mathrm{C}$.

Figure 5 Shear rheometer data of complex viscosity for filaments containing $30 \%$ theophylline, $35 \%$ PEG 6K, and 35\% PEO (100K, 200K, 300K, 600K, and 900K) at A) 110 and B) $145^{\circ} \mathrm{C}$.

Figure 6 Shear rheometer data of storage modulus and loss modulus for filaments containing $30 \%$ theophylline, $35 \%$ PEG $6 \mathrm{~K}$, and $35 \%$ PEO $(100 \mathrm{~K}, 200 \mathrm{~K}, 300 \mathrm{~K}, 600 \mathrm{~K}$, and $900 \mathrm{~K})$ at A) 110 and B) $145^{\circ} \mathrm{C}$.

400

Figure 7 (A1) Rendered image and (A2) photograph of radiator-like design. (B1) Top view , (B 2) side view and (B3) photograph of radiator-like doses based on theophylline :PEG 6K:PEO 600K 30:35:35.

Figure 8 In vitro release pattern of: A) $0.6 \mathrm{~mm}, 1.0 \mathrm{~mm}, 1.5 \mathrm{~mm}$, and $2.0 \mathrm{~mm}$ spaced radiatorlike 3D printed tablet containing $30 \%$ theophylline, 35\% PEG 6K, and 35\% PEO 600K, and B) tablets prepared using filaments composed of $30 \%$ theophylline, 35\% PEG 6K, and 35\%

\section{List of Tables}

407

Table 1 Composition, processing temperatures and FDM 3D printing compatibility of theophylline filament based on PEO with different molecular weights.

409 Table 2 Solubility parameter and its components of theophylline and PEO/PEG in $\mathrm{MPa}^{1 / 2}$.

$410 \quad$ Table 3

\section{Supplementary Data}

413 Figure S1a XRPD patterns of $30 \%$ theophylline, 35\% PEG 6K, and 35\% PEO (100K, $414200 \mathrm{~K}, 300 \mathrm{~K}, 600 \mathrm{~K}$, and $900 \mathrm{~K}$.

415 Figure S1b XRPD patterns of: A) raw theophylline, raw PEG 6K, raw PEO 100K, and 416 filament containing 30:35:35 theophylline:PEG 6K:PEO 100K, B) raw theophylline, raw 417 PEG 6K, raw PEO 200K and 30:35:35 theophylline:PEG 6K:PEO 200K. 
418 Figure S1c XRPD patterns of raw theophylline, raw PEG 6K, raw PEO 300K, and filament 419 containing 30:35:35 theophylline:PEG 6K:PEO 300K.

420 Figure S1d XRPD patterns of raw theophylline, raw PEG 6K, raw PEO 600K, and filament 421 containing 30:35:35 theophylline:PEG 6K:PEO 600K.

422 Figure S1e XRPD patterns of raw theophylline, raw PEG 6K, raw PEO 900K, and filament 423 containing 30:35:35 theophylline:PEG 6K:PEO 900K.

424 Figure S2 In vitro release pattern of A) theophylline from FDM 3D printed caplet tablet and a 425 radiator-like dose with spacing of $2 \mathrm{~mm}$ (theophylline: PEG 6K: PEO 600K 30:35:35). 